Braz J Med Biol Res, September 2012, Volume 45(9) 834-840

doi: 10.1590/S0100-879X2012007500120

Caffeine intake by patients with autosomal dominant polycystic kidney disease

L.C. Vendramini, J.L. Nishiura, A.C. Baxmann and I.P. Heilberg

The Brazilian Journal of Medical and Biological Research is partially financed by

\section{Q}

da Ciência e Tecnologia

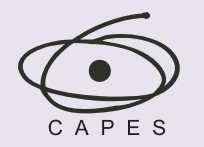

Ministério da Educação
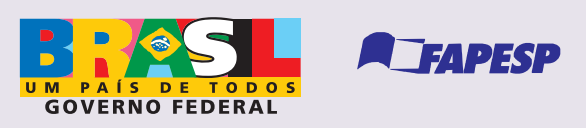

Institutional Sponsors
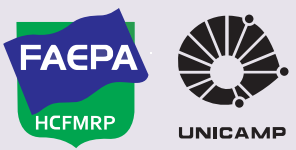

Ф SHIMADZU UNICAMP
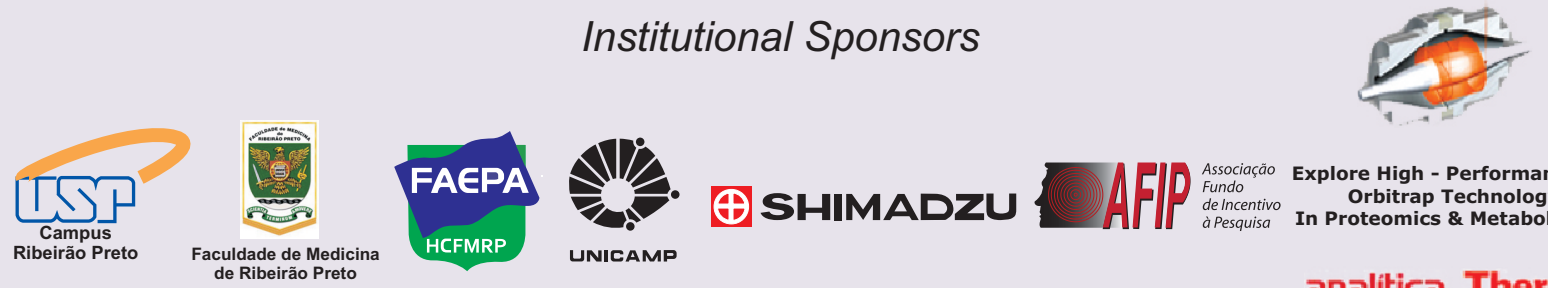

lore High - Performance MS Orbitrap Technology In Proteomics \& Metabolomics \section{analítica}




\title{
Caffeine intake by patients with autosomal dominant polycystic kidney disease
}

\author{
L.C. Vendramini, J.L. Nishiura, A.C. Baxmann and I.P. Heilberg \\ Disciplina de Nefrologia, Departamento de Medicina, \\ Universidade Federal de São Paulo, São Paulo, SP, Brasil
}

\begin{abstract}
Because caffeine may induce cyst and kidney enlargement in autosomal dominant polycystic kidney disease (ADPKD), we evaluated caffeine intake and renal volume using renal ultrasound in ADPKD patients. Caffeine intake was estimated by the average of 24-h dietary recalls obtained on 3 nonconsecutive days in 102 ADPKD patients (68 females, 34 males; $39 \pm 12$ years) and compared to that of 102 healthy volunteers (74 females, 28 males; $38 \pm 14$ years). The awareness of the need for caffeine restriction was assessed. Clinical and laboratory data were obtained from the medical records of the patients. Mean caffeine intake was significantly lower in ADPKD patients versus controls (86 vs $134 \mathrm{mg} /$ day), and 63\% of the ADPKD patients had been previously aware of caffeine restriction. Caffeine intake did not correlate with renal volume in ADPKD patients. There were no significant differences between the renal volumes of patients in the highest and lowest tertiles of caffeine consumption. Finally, age-adjusted multiple linear regression revealed that renal volume was associated with hypertension, chronic kidney disease stage 3 and the time since diagnosis, but not with caffeine intake. The present small cross-sectional study indicated a low level of caffeine consumption by ADPKD patients when compared to healthy volunteers, which was most likely due to prior awareness of the need for caffeine restriction. Within the range of caffeine intake observed by ADPKD patients in this study (0-471 mg/day), the renal volume was not directly associated with caffeine intake.
\end{abstract}

Key words: Polycystic kidneys; ADPKD; Cyclic AMP; Caffeine; Nutrition; Renal volume

\section{Introduction}

Autosomal dominant polycystic kidney disease (ADPKD) is the most common inherited renal cystic disease and is characterized by the development of renal cysts and various extrarenal manifestations. A disruption of normal polycystic function by mutations in polycystic kidney disease 1 (PKD1) or polycystic kidney disease 2 (PKD2) genes predisposes the individual to cyst formation through the loss of mechanical cues in the tubular epithelial cells that regulate tissue morphogenesis (1).

ADPKD patients may remain asymptomatic for years or present signs and symptoms of flank pain, hematuria, urinary tract infections, hypertension and nephrolithiasis (2). Multiple cysts generally increase in size and number with age, ultimately resulting in end-stage renal disease. Magnetic resonance imaging (MRI) data from an observational trial of ADPKD showed that kidney volume increases by an average of $5.3 \%$ per year but with a wide range of variability, and that renal volume can be used to monitor disease progression before a measurable decline in function (3).
Currently, there are no established treatments to slow the progression of the disease, but several promising therapeutic options are being tested in ongoing clinical trials (1). Arginine vasopressin (AVP) is the major stimulus that leads to an increase in intracellular cyclic adenosine monophosphate (cAMP) levels in cyst epithelial cells, contributing to cyst and kidney enlargement (1). The administration of AVP V2 receptor antagonists inhibits the development of polycystic kidney disease in animal models, and clinical studies have been initiated with Tolvaptan (4). Similarly, suppressing AVP release with high water intake is protective in rats (5) and is a promising alternative approach in humans (6).

Receptor-mediated agents that increase the activity of adenyl cyclase and inhibitors of phosphodiesterase (PDE) such as caffeine, a 1,3,7-trimethylxanthine (7), have the potential to accelerate the progression of ADPKD. In a very elegant experimental study conducted by Belibi et al. (8), a $\mathrm{V} 2$ receptor agonist (DDAVP) increased the intracellular

Correspondence: I.P. Heilberg, Disciplina de Nefrologia, Departamento de Medicina, UNIFESP, Rua Botucatu, 740, 04023-900 São Paulo, SP, Brasil. Fax: +55-11-5904-1684. E-mail: ipheilberg@nefro.epm.br

Received December 7, 2011. Accepted July 4, 2012. Available online July 20, 2012. Published August 17, 2012. 
levels of cAMP in the cyst epithelial cells, and this effect was further increased by caffeine concentrations in a clinically relevant range. Subsequently, chronic caffeine administration to Han:SPRD rats was shown to exacerbate hypertension in this animal model, but no effect was observed on glomerular filtration rate (GFR) or cyst development (9). Although it has been recommended that ADPKD patients reduce their caffeine intake based on these findings, studies in humans are needed.

In the present study, we determined the consumption of caffeine by ADPKD patients through 24-h dietary recalls and discriminated among its various dietary sources. In addition, we sought associations between caffeine intake and clinical and laboratory data.

\section{Material and Methods}

A total of 102 adult patients evaluated at the Nephrology Division of Universidade Federal de São Paulo, who had a diagnosis of ADPKD because of an affected parent or sibling with ADPKD and renal cysts detected by ultrasound using the diagnostic criteria of Pei et al. (10), were selected to participate in the study. One hundred and two age-matched healthy volunteers with similar body mass indexes (BMIs) were recruited as controls. The Local Ethics Committee approved the study and written informed consent was obtained from all participants.

\section{Assessment of dietary habits and anthropometric parameters}

All participants were submitted to anthropometric assessment that included body weight and height measurements, as well as BMI calculation as weight $(\mathrm{kg}) / \mathrm{height}\left(\mathrm{m}^{2}\right)$. A complete investigation of nutrient consumption, including caffeine, was conducted using a $24-\mathrm{h}$ dietary recall (24R)

Table 1. Caffeine content of different foods.

\begin{tabular}{llc}
\hline Caffeine-containing foods & $\begin{array}{c}\text { Household } \\
\text { measurement }\end{array}$ & $\begin{array}{c}\text { Amount of } \\
\text { caffeine }(\mathrm{mg})\end{array}$ \\
\hline Coffee (espresso) & 1 cup $(60 \mathrm{~mL})$ & 59.8 \\
Coffee (instant) & 1 cup $(60 \mathrm{~mL})$ & 44.1 \\
Coffee (brewed) & 1 cup $(60 \mathrm{~mL})$ & 36.5 \\
Black tea & 1 cup $(150 \mathrm{~mL})$ & 34.6 \\
Cola soft drinks & 1 can $(350 \mathrm{~mL})$ & 30.9 \\
Semisweet chocolate & 1 bar $(30 \mathrm{~g})$ & 26.4 \\
Dark sweet chocolate & 1 bar $(30 \mathrm{~g})$ & 11.4 \\
Yerba mate tea & 1 cup $(150 \mathrm{~mL})$ & 10.3 \\
White chocolate & 1 bar $(30 \mathrm{~g})$ & 5.7 \\
Chocolate powder & 1 tablespoon & 3.7 \\
Guarana soft drinks & 1 can $(350 \mathrm{~mL})$ & 3.8 \\
\hline
\end{tabular}

Data taken from Camargo et al. $(11,12)$. on three nonconsecutive days ( 2 weekdays and 1 weekend day). The average of these $24 \mathrm{R}$ was used as an estimate of the intake. A trained interviewer (one of the authors, LCV) collected the food intake data and showed visual aids to assist the participants in estimating the amount consumed. Following the last 24R, the ADPKD patients were asked whether they had ever read or heard (from health care practitioners, friends, relatives, or patients with the same disease) about any dietary advice for polycystic kidney disease. To avoid suggestibility, only patients who spontaneously answered, "Yes, l've read or been told to reduce my intake of caffeine-containing foods", were considered to be "aware". All others were classified as "unaware". The daily energy and macronutrient intakes were calculated with a computerized program developed in our department. The food composition table used in the program was from the US Department of Agriculture. Caffeine intake was calculated according to data from Camargo et al. $(11,12)$, which quantified the caffeine content in Brazilian food, including coffee (instant, brewed and espresso), regular or diet soft drinks (cola or guarana), teas (yerba mate and black) and chocolate bars (dark, white and semi-sweet), as shown in Table 1. Because the protein intake or ash content of the diet may accelerate the course of ADPKD (13), the potential renal acid load (PRAL), an established method for estimating the acid load of diets, was determined according to the protein, phosphorus, magnesium, potassium, and calcium dietary contents using the formula proposed by Frassetto et al. (14).

Clinical and laboratory parameters were obtained from the medical records of ADPKD patients. Hypertension was considered to be present on the basis of a history of hypertension, blood pressure measurements and use of antihypertensive medications. The renal volume was measured by ultrasound and calculated based on kidney length, width and anteroposterior diameter using a modified ellipsoid formula (15). The values for both kidneys were combined to yield the total kidney volume (3). Serum creatinine was determined using a modified Jaffé reaction (16) and a Hitachi 912 analyzer (Roche Diagnostic System, Switzerland) based on an isotope dilution mass spectrometry-traceable method. The estimated GFR (eGFR) was obtained using the re-expressed, four-variable modification of diet in renal disease (MDRD) study equation (13). The stages of chronic kidney disease (CKD) were defined according to KDIGO (Kidney Disease: Improving Global Outcomes) guidelines (17).

\section{Statistical analysis}

Parametric variables are reported as means \pm SD and nonparametric variables as medians and interquartile ranges. The control and ADPKD groups were compared by the $t$-test. Caffeine intake was divided into tertiles. The clinical and laboratory parameters were compared by oneway analysis of variance (ANOVA) or the chi-square test 
(categorical variables). Pearson's correlation coefficient was used to determine the correlation between caffeine intake and renal volume or eGFR (unadjusted and adjusted for age). A univariate linear analysis was used to reduce the pool of variables entered into the multiple regression analysis. The independent variables were age, caffeine intake, time since diagnosis, presence of hypertension, CKD stages $1 / 2$ or stage 3 , dietary protein intake, and PRAL. A stepwise multiple linear regression analysis adjusted for age was performed to describe the association between the renal volume or eGFR and the statistically significant explanatory variables in the model. For the purpose of this analysis, because of the absence of a normal distribution, the renal volume, eGFR, caffeine intake, protein, and time since diagnosis were converted to a logarithmic $(\log 10)$ scale. The level of significance was set at $\mathrm{P}<0.05$.

\section{Results}

A total of 102 ADPKD patients (68 women and 34 men) and 102 healthy individuals ( 74 women and 28 men) participated in this study. Table 2 shows that ADPKD and control groups did not differ in mean age, BMI or daily intakes of energy, proteins, carbohydrates, and lipids. The mean PRAL values did not differ significantly between the ADPKD and control groups. However, the mean caffeine intake was significantly lower in ADPKD patients than in controls (86 \pm 77 vs $134 \pm 116 \mathrm{mg} /$ day, $\mathrm{P} \leq 0.001)$. As shown in Table 3 , coffee most commonly contributed to the caffeine intake in all groups, followed by soft drinks, chocolate products and teas. There was no correlation between the level of caffeine intake and renal volume $(r=0.127 ; P=0.200)$ or eGFR ( $r=0.07 ; P=0.445)$ in the entire sample of ADPKD patients. The lack of a correlation between caffeine intake and renal volume $(r=0.015 ; P=0.879)$ or eGFR $(r=0.065$; $\mathrm{P}=0.520$ ) persisted even after adjusting for age, as depicted in Figure $1 \mathrm{~A}$ and $\mathrm{B}$.

The clinical and laboratorial characteristics of ADPKD patients according to the tertiles of caffeine consumption are presented in Table 4. The age, time since diagnosis, level of renal function, percentage of hypertensive patients, and renal volume did not differ significantly across the tertiles of caffeine intake. Sixty-four of the 102 ADPKD patients were considered to have been previously aware of the need for caffeine restriction. The unaware ADPKD patients consumed significantly more caffeine than the aware patients $(113 \pm 92$ vs $69 \pm 60 \mathrm{mg} /$ day, $P=0.001)$. Because a formal recommendation for caffeine restriction had not been given to the present series, we estimated the time of prior medical care provided by either a general practitioner or a nephrologist. Although there was a trend towards a higher prevalence of aware patients (82\%) with a longer mean estimated time of medical follow-up (39.5 \pm 24 months) among the patients in the first tertile of caffeine intake (0-41.6 mg), there was no significant difference
Table 2. Anthropometric and nutritional parameters of patients and control subjects.

\begin{tabular}{lcc}
\hline Parameters & $\begin{array}{c}\text { Controls } \\
(\mathrm{N}=102)\end{array}$ & $\begin{array}{c}\text { ADPKD } \\
(\mathrm{N}=102)\end{array}$ \\
\hline Gender (F/M) & $74 / 28$ & $68 / 34$ \\
Age (years) & $38 \pm 14$ & $39 \pm 12$ \\
BMI (kg/m $\left.{ }^{2}\right)$ & $24.8 \pm 4.8$ & $25.7 \pm 4.5$ \\
Energy (kcal/day) & $1966 \pm 601$ & $2018 \pm 608$ \\
Protein intake $\left(\mathrm{g} \cdot \mathrm{kg}^{-1} \cdot\right.$ day $\left.^{-1}\right)$ & $1.2 \pm 0.3$ & $1.2 \pm 0.4$ \\
PRAL (mEq/day) & $20 \pm 14$ & $21 \pm 14$ \\
Carbohydrate intake (g/day) & $246 \pm 78$ & $268 \pm 82$ \\
Lipid intake (g/day) & $70 \pm 26$ & $68 \pm 25$ \\
Caffeine intake (mg/day) & $134 \pm 116$ & $86 \pm 77^{*}$ \\
\hline
\end{tabular}

Data are reported as means \pm SD. ADPKD $=$ autosomal dominant polycystic kidney disease patients; $\mathrm{BMI}=$ body mass index; PRAL $=$ potential renal acid load. ${ }^{*} \mathrm{P}=0.001$ compared to controls ( $t$-test).

Table 3. Mean daily caffeine intake from the main dietary sources.

\begin{tabular}{lcc}
\hline Groups & Controls $(\mathrm{N}=102)$ & ADPKD $(\mathrm{N}=102)$ \\
\hline Coffee & $115.9 \pm 117.9^{*}$ & $74.9 \pm 74.5^{*}$ \\
Soft drinks & $12.5 \pm 21.1$ & $6.9 \pm 11.2$ \\
Chocolate products & $3.9 \pm 4.6$ & $2.1 \pm 4.2$ \\
Teas & $1.8 \pm 5.1$ & $1.6 \pm 6.3$ \\
\hline
\end{tabular}

Data are reported in $\mathrm{mg}$ as means $\pm \mathrm{SD}$. Groups: coffee (espresso, instant and brewed); soft drinks (cola and guarana); chocolate products (chocolate powder, dark, semi-sweet, and white chocolates); teas (mate and black). ADPKD = autosomal dominant polycystic kidney disease patients. ${ }^{*} \mathrm{P}<0.05$ compared to soft drinks, chocolate products and teas ( $t$-test).

when compared to the percentage of aware patients and their respective mean time of medical follow-up within the second $(56 \%)$ and third (50\%) tertiles $(29.5 \pm 22.5$ and $29.8 \pm 23$ months).

In a separate analysis of a small number of patients $(\mathrm{N}=17)$ whose medical records contained an additional ultrasound obtained at the time of diagnosis of PKD, we observed a $32 \%$ mean increase in renal volume, and the median values were significantly different (559.6 vs 307.4 $\mathrm{mL}, \mathrm{P}<0.001$ ) between the two ultrasounds (mean interval of $49 \pm 19$ months). However, when this small fraction of patients was classified according to a caffeine consumption above or below the median caffeine consumption level ( $85.9 \mathrm{mg} /$ day), the patients who consumed less than 85.9 $\mathrm{mg} /$ day $(\mathrm{N}=8)$ did not show a significant difference in the mean percentage of increment of renal volume compared to patients with a caffeine consumption $\geq 85.9 \mathrm{mg} /$ day $(\mathrm{N}$ 
= 9) $(31$ vs 35\%, P = 0.681).

Univariate analysis of all ADPKD patients showed that age, hypertension, CKD stage 3 and time since diagnosis were significant variables for an association with renal volume; however, caffeine intake, protein intake and PRAL were not significant (data not shown). Stepwise multiple linear regression adjusted for age revealed that the only independent variables associated with renal volume were the presence of hypertension $(P=0.025), C K D$ stage 3 ( $P$ $<0.001)$ and the time since diagnosis $(P=0.035)$.

Another univariate analysis performed with eGFR as the dependent variable revealed that age, hypertension and protein intake, but not caffeine intake, were associated with eGFR (data not shown). However, in the stepwise multiple linear regression adjusted for age, the only variable associated with eGFR was the presence of hypertension $(P=0.003)$.

\section{Discussion}

Given that caffeine is responsible for PDE inhibition, leading to increased levels of cAMP and activating the extracellular signal-regulated kinase (ERK) pathway with consequent increases in cell proliferation and fluid secretion (18) in ADPKD cystic epithelium, patients with ADPKD are being advised to reduce their caffeine consumption (8). Data from a cell culture study that showed the effects of caffeine on the accumulation of cAMP in cells retrieved from renal cysts of ADPKD patients strongly support such recommendations (8). However, despite the increases in arterial blood pressure induced by chronic caffeine intake in a non-orthologous animal model of PKD, no effect on cyst development was observed (9). To our knowledge, human studies focusing on the caffeine consumption by ADPKD patients have not yet been reported.
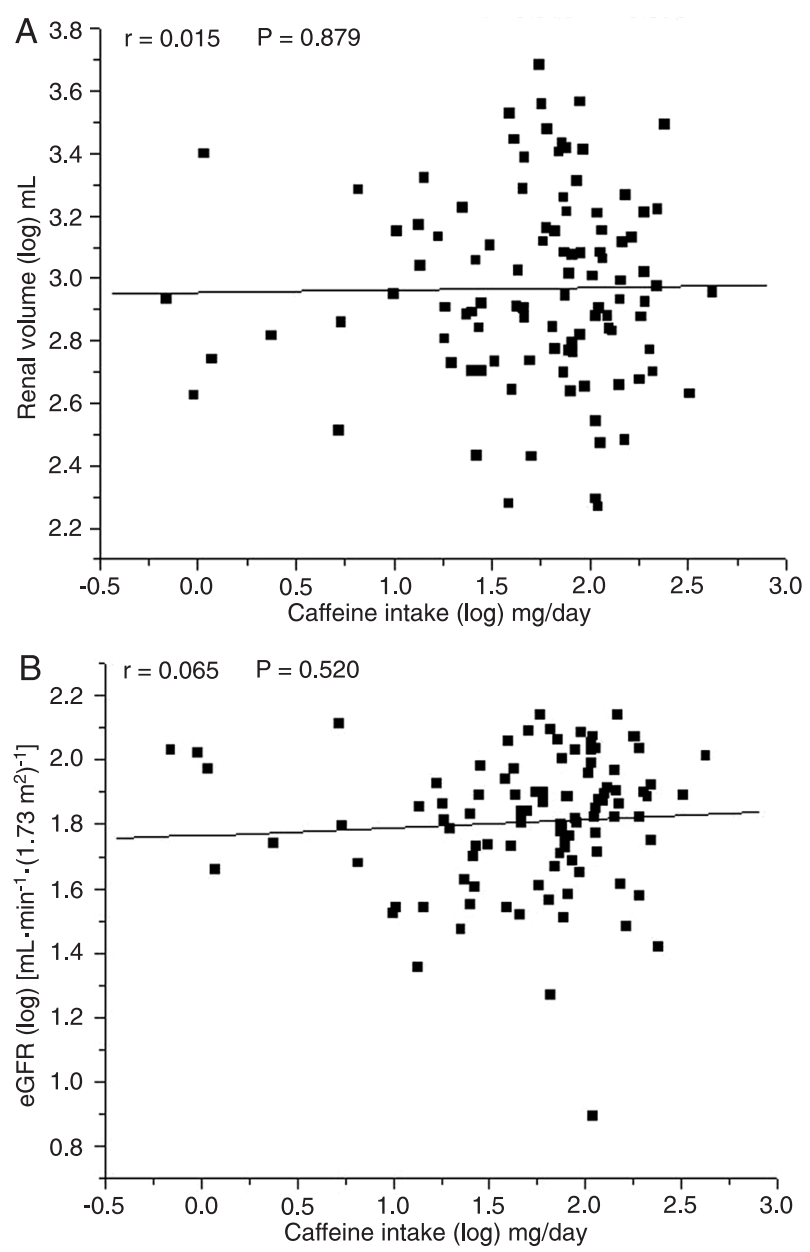

Figure 1. $A$, Pearson's correlation between log caffeine intake and log renal volume. $B$, Pearson's correlation between log caffeine intake and log estimated glomerular filtration rate (eGFR).

Table 4. Clinical and laboratory characteristics according to caffeine intake.

\begin{tabular}{lccc}
\hline Parameters & \multicolumn{3}{c}{ Caffeine intake } \\
\cline { 2 - 4 } & $0-41.6 \mathrm{mg} /$ day $(\mathrm{N}=34)$ & $41.7-98.7 \mathrm{mg} / \mathrm{day}(\mathrm{N}=34)$ & $98.8-471.4 \mathrm{mg} / \mathrm{day}(\mathrm{N}=34)$ \\
\hline Age (years) & $36 \pm 12$ & $39 \pm 13$ & $43 \pm 11$ \\
Time since diagnosis (months) & $112 \pm 103$ & $99 \pm 88$ & $101 \pm 99$ \\
CKD1/2 (N, \%) & $21(62)$ & $19(56)$ & $23(68)$ \\
CKD3 (N, \%) & $13(38)$ & $15(44)$ & $11(32)$ \\
Hypertension (N, \%) & $16(47)$ & $26(76)$ & $22(65)$ \\
Serum creatinine $(\mathrm{mg} / \mathrm{dL})$ & $1.3 \pm 0.73$ & $1.3 \pm 0.7$ & $1.4 \pm 1.2$ \\
eGFR [mL·min-1.(1.73 $\left.\left.\mathrm{m}^{2}\right)^{-1}\right]$ & $74 \pm 33$ & $74 \pm 35$ & $73 \pm 35$ \\
Renal volume $(\mathrm{mL})$ & $787(306-3848)$ & $1100(260-5517)$ & $746(219-3588)$ \\
\hline
\end{tabular}

Parametric variables are reported as means \pm SD. Renal volume (nonparametric variable) is reported as median (25th to 75th percentile). CKD1/2 = chronic kidney disease stages 1/2; CKD3 = chronic kidney disease stage 3; eGFR = estimated glomerular filtration rate. No statistically significant differences were detected among tertiles (ANOVA or chi-square test for categorical variables). 
Our results showed a low mean caffeine intake by ADPKD patients $(85.7 \mathrm{mg} /$ day), which was most likely due to the awareness of a need for caffeine restriction by most of the patients (63\%). Since a food history may yield imprecise data because this method measures only particular days and relies on memory, a 5-step method developed by the USDA for collecting $24 \mathrm{R}$ was used in the current study to minimize misreporting (19). The trend of higher prevalence of aware patients $(82 \%)$ in the first tertile of caffeine consumption further supports the view that awareness contributed to low caffeine consumption. We observed that these patients also had a longer previous medical follow-up. However, half of the patients belonging to the second and third tertiles were aware of caffeine restriction recommendations. These findings suggest that not all patients who received some previous dietary advice adhered to it. Nevertheless, the aim of the present study was not to evaluate compliance, considering that we were not the ones to provide dietary recommendations. We hypothesized whether or not "awareness" could interfere with the results obtained from the food recalls, and we indeed found out that aware patients consumed even less caffeine than unaware patients. It must be emphasized that the caffeine consumption level was not too high even in the third tertile group, where only three patients reported intake of more than $300 \mathrm{mg} /$ day.

The mean daily caffeine consumption by healthy subjects in the present series was somewhat lower $(134 \mathrm{mg} /$ day) than reports in the Brazilian population ( $171 \mathrm{mg} /$ day) (13) and much lower than the estimates from other countries, such as the United States (200 mg/day), United Kingdom (280 mg/day) and Denmark (490 mg/day) (20).

In the present series, coffee consumption accounted for most of the daily caffeine intake in all groups. These findings agree with those for the Brazilian population (12) and with American data $(20,21)$, which show that the major contributor to caffeine intake in the US population is coffee. Conversely, the major source of caffeine intake in the UK is tea (20).

The other purpose of the present study was to determine the association between caffeine intake and the clinical and laboratory findings in this sample of ADPKD patients. An age-adjusted correlation between caffeine intake and renal volume was not found in the ADPKD sample. ADPKD patients in the highest tertile of caffeine consumption did not exhibit a higher median renal volume than the other two tertiles. Although the purpose of this cross-sectional study was not to verify the impact on disease progression, it is well established that renal volume increases with age and is directly associated with hypertension, ultimately leading to a progressive loss of renal function in the ADPKD setting (3). Therefore, a univariate analysis was performed to isolate the significant dependent variables, and a multivariate linear regression was then used to adjust the bias of these interfering factors in kidney enlargement. The stepwise multiple linear regression adjusted for age showed that renal volume was associated with hypertension, CKD stage 3 and the time since diagnosis but not with caffeine intake. Given that the protein or ash content of diets has been proposed to accelerate the course of ADPKD, we evaluated the impact of protein intake and PRAL, which provides an estimate of the production of endogenous acid that exceeds the level of alkali produced for given amounts of foods ingested daily, on kidney volume and eGFR (14). Because negative values indicate alkaline foods and positive values indicate that the food item has an acid load, high-protein diets are usually considered high-PRAL diets. Protein intake and PRAL were not associated with kidney volume or with eGFR in the present age-adjusted multiple regression analysis. Because the main purpose of the study was to evaluate the caffeine intake, we did not focus on previous instructions regarding protein restriction. Therefore, we could not precisely quantify how many patients were following such recommendations. The mean protein intake of $1.2 \mathrm{~g} \mathrm{~kg}^{-1}$. day ${ }^{-1}$ (Table 2) certainly shows that patients did not reduce their protein intake. However, it is worth mentioning that most patients $(62 \%)$ had a wellpreserved renal function.

The low level of caffeine consumption found in these ADPKD patients might have contributed to the lack of correlation between caffeine intake and renal volume. Although the results from cell culture studies cannot be compared to our clinical observations, Belibi et al. (8) showed that 10 to $50 \mu \mathrm{M}$ caffeine potentiated the effects of desmopressin increasing cAMP levels in ADPKD cells. According to these investigators, pharmacokinetic studies indicate that the ingestion of 1 to 3 cups of coffee leads to plasma caffeine levels between 10 and $50 \mu \mathrm{M}$; therefore, this amount was considered clinically relevant. Because higher levels of caffeine would be expected with greater amounts of caffeine, leading to greater elevations of renal tissue cAMP, further experiments by these investigators used amounts up to $1000 \mu \mathrm{M}$. However, one can argue that such amounts are far above the typical human intake. Extrapolations from animal studies may not be precise, but the caffeine amount that, according to Tanner et al. (9), exacerbated hypertension but did not change the GFR in Han:SPRD rats was equivalent to a human intake of approximately 4 cups of coffee per day (380 mg caffeine). In our series, in the range of caffeine intake presently disclosed, there was no correlation between caffeine intake and age-adjusted eGFR. In addition, the multiple regression analysis revealed that hypertension (but not caffeine intake) was the only variable associated with the age-adjusted eGFR in ADPKD patients. In the aforementioned study (9), greater doses of caffeine, equivalent to 9 cups of coffee per day (765 mg caffeine), which are far above the human intake, also increased the mean arterial pressure with a trend toward a lower GFR but did not result in more or larger kidney cysts in this PKD model (9). Because caffeine is a weak phosphodiesterase 
inhibitor (22) and plasma caffeine levels achieved after coffee drinking are much lower than those needed to affect cAMP breakdown, these investigators (9) proposed the possibility that caffeine might have affected this disease through its effects on arterial pressure and not by changing cAMP, although cAMP was not measured in their study.

One potential limitation of our study is the measurement of kidney volume. We are aware that sonography is highly operator dependent, lacks accuracy to detect small changes in renal volume (23) and that the renal volume measured by MRI is more appropriate $(24,25)$, especially when the goal is to determine the renal blood flow and progression, which was not the focus of the present study. However, MRI is not employed in ADPKD patients routinely because of its high cost. Despite ultrasound limitations, other investigators have shown that determining renal volume by this method $(26,27)$ may also be a useful marker for the progression of ADPKD.

The cross-sectional nature and sample size of this study may be another limitation. Because of the worldwide differences in caffeine consumption, the methods of preparation (regular, espresso or instant), cup sizes and other peculiarities, scientific information about caffeine intake in different populations is still warranted. Moreover, due to the lack of human data linking coffee intake to worsening of ADPKD,

\section{References}

1. Torres VE. Treatment strategies and clinical trial design in ADPKD. Adv Chronic Kidney Dis 2010; 17: 190-204.

2. Nishiura JL, Neves RF, Eloi SR, Cintra SM, Ajzen SA, Heilberg IP. Evaluation of nephrolithiasis in autosomal dominant polycystic kidney disease patients. Clin J Am Soc Nephrol 2009; 4: 838-844.

3. Grantham JJ, Torres VE, Chapman AB, Guay-Woodford LM, Bae KT, King BF Jr, et al. Volume progression in polycystic kidney disease. N Engl J Med 2006; 354: 2122-2130.

4. Torres VE. Role of vasopressin antagonists. Clin J Am Soc Nephrol 2008; 3: 1212-1218.

5. Nagao S, Nishii K, Katsuyama M, Kurahashi H, Marunouchi $\mathrm{T}$, Takahashi $\mathrm{H}$, et al. Increased water intake decreases progression of polycystic kidney disease in the PCK rat. $J$ Am Soc Nephrol 2006; 17: 2220-2227.

6. Barash I, Ponda MP, Goldfarb DS, Skolnik EY. A pilot clinical study to evaluate changes in urine osmolality and urine cAMP in response to acute and chronic water loading in autosomal dominant polycystic kidney disease. Clin J Am Soc Nephrol 2010; 5: 693-697.

7. Heckman MA, Weil J, Gonzalez de ME. Caffeine $(1,3$, 7-trimethylxanthine) in foods: a comprehensive review on consumption, functionality, safety, and regulatory matters. $J$ Food Sci 2010; 75: R77-R87.

8. Belibi FA, Wallace DP, Yamaguchi T, Christensen M, Reif G, Grantham JJ. The effect of caffeine on renal epithelial cells from patients with autosomal dominant polycystic kidney disease. J Am Soc Nephrol 2002; 13: 2723-2729.

9. Tanner GA, Tanner JA. Chronic caffeine consumption exac- observational studies to identify associations between caffeine intake with either cysts or renal enlargement in a clinical setting should be replicated in larger samples. Additionally, the impact of caffeine intake on disease progression was not addressed here.

Nevertheless, several important points must be considered. Conducting a randomized clinical trial to address the role of caffeine restriction in ADPKD seems difficult because including a non-restricted control group would be considered unethical. Thus far, hypothesis-generating experimental studies have already been integrated into clinical practice. The premature incorporation of a caffeine restriction policy renders longitudinal studies even more difficult to perform because awareness has already spread to patients and non-health professionals.

In conclusion, within the range of caffeine consumption presently evaluated and considering the limitations of a small cross-sectional study, the food-history methodology and ultrasound as a method for renal volume measurement, no correlation was observed between caffeine intake and renal volume or eGFR in ADPKD patients.

\section{Acknowledgments}

Research supported by CAPES. erbates hypertension in rats with polycystic kidney disease. Am J Kidney Dis 2001; 38: 1089-1095.

10. Pei Y, Obaji J, Dupuis A, Paterson AD, Magistroni R, Dicks $E$, et al. Unified criteria for ultrasonographic diagnosis of ADPKD. J Am Soc Nephrol 2009; 20: 205-212.

11. Camargo MCR, Toledo MC. HPLC determination of caffeine in tea, chocolate products and carbonated beverages. J Sci Food Agric 1999; 79: 1861-1864.

12. Camargo MC, Toledo MC, Farah HG. Caffeine daily intake from dietary sources in Brazil. Food Addit Contam 1999; 16: 79-87.

13. Levey AS, Bosch JP, Lewis JB, Greene T, Rogers N, Roth D. A more accurate method to estimate glomerular filtration rate from serum creatinine: a new prediction equation. Modification of Diet in Renal Disease Study Group. Ann Intern Med 1999; 130: 461-470.

14. Frassetto LA, Lanham-New SA, Macdonald HM, Remer T, Sebastian A, Tucker KL, et al. Standardizing terminology for estimating the diet-dependent net acid load to the metabolic system. J Nutr 2007; 137: 1491-1492.

15. Schrier RW, McFann KK, Johnson AM. Epidemiological study of kidney survival in autosomal dominant polycystic kidney disease. Kidney Int 2003; 63: 678-685.

16. Bartels $\mathrm{H}$, Bohmer M, Heierli C. [Serum creatinine determination without protein precipitation]. Clin Chim Acta 1972; 37: 193-197.

17. Levey AS, Eckardt KU, Tsukamoto Y, Levin A, Coresh J, Rossert J, et al. Definition and classification of chronic kidney disease: a position statement from Kidney Disease: 
Improving Global Outcomes (KDIGO). Kidney Int 2005; 67: 2089-2100.

18. Belibi FA, Reif G, Wallace DP, Yamaguchi T, Olsen L, Li H, et al. Cyclic AMP promotes growth and secretion in human polycystic kidney epithelial cells. Kidney Int 2004; 66: 964973.

19. Moshfegh AJ, Rhodes DG, Baer DJ, Murayi T, Clemens JC, Rumpler WV, et al. The US Department of Agriculture Automated Multiple-Pass Method reduces bias in the collection of energy intakes. Am J Clin Nutr 2008; 88: 324-332.

20. Barone JJ, Roberts HR. Caffeine consumption. Food Chem Toxicol 1996; 34: 119-129.

21. Frary $C D$, Johnson RK, Wang MQ. Food sources and intakes of caffeine in the diets of persons in the United States. J Am Diet Assoc 2005; 105: 110-113.

22. Mangoo-Karim R, Uchic ME, Grant M, Shumate WA, Calvet JP, Park $\mathrm{CH}$, et al. Renal epithelial fluid secretion and cyst growth: the role of cyclic AMP. FASEB J 1989; 3: 26292632.

23. O'Neill WC, Robbin ML, Bae KT, Grantham JJ, Chapman $A B$, Guay-Woodford LM, et al. Sonographic assessment of the severity and progression of autosomal dominant polycystic kidney disease: the Consortium of Renal Imaging
Studies in Polycystic Kidney Disease (CRISP). Am J Kidney Dis 2005; 46: 1058-1064.

24. Torres VE, King BF, Chapman AB, Brummer ME, Bae KT, Glockner JF, et al. Magnetic resonance measurements of renal blood flow and disease progression in autosomal dominant polycystic kidney disease. Clin J Am Soc Nephrol 2007; 2: 112-120.

25. Chapman AB, Guay-Woodford LM, Grantham JJ, Torres VE, Bae KT, Baumgarten DA, et al. Renal structure in early autosomal-dominant polycystic kidney disease (ADPKD): The Consortium for Radiologic Imaging Studies of Polycystic Kidney Disease (CRISP) cohort. Kidney Int 2003; 64: 10351045.

26. Grantham JJ, Chapman AB, Torres VE. Volume progression in autosomal dominant polycystic kidney disease: the major factor determining clinical outcomes. Clin J Am Soc Nephrol 2006; 1: 148-157.

27. Fick-Brosnahan GM, Belz MM, McFann KK, Johnson AM, Schrier RW. Relationship between renal volume growth and renal function in autosomal dominant polycystic kidney disease: a longitudinal study. Am J Kidney Dis 2002; 39: $1127-1134$ 